\title{
Daños relacionados con el consumo de cocaína: la punta del iceberg es lo que vemos
}

\author{
César Pereiro Gómez*; Ana Bermejo Barrera** \\ * Psiquiatra. Director Médico de la Unidad Asistencial de Drogodependencias de A Coruña (ACLAD). Plan de Galicia sobre Drogas. \\ ** Catedrática de Toxicología. Facultad de Medicina. Universidad de Santiago de Compostela.

\section{RESUMEN}

Según el Observatorio Europeo de las Drogas y Toxicomanías, la cocaína es, después del cannabis, la droga ilegal más consumida en Europa. La percepción sobre los daños relacionados con el consumo de esta sustancia son claramente percibidos en algunas áreas mientras en otras pueden pasar inadvertidos, infravalorados o mal dimensionados. Los sistemas de información existentes con toda seguridad ofrecen deficiencias al valorar la morbimortalidad relacionada con la cocaína. Además del potencial que la cocaína tiene para producir daños directos en distintos órganos, su consumo puede condicionar graves perjuicios a través de otras circunstancias que no siempre se relacionan con esta droga: homicidios, accidentes de tráfico y laborales, adquisición de enfermedades infecciosas relacionadas con conductas de riesgo, suicidios, etc. La cocaína, está cada vez más presente en episodios de violencia tales como peleas o agresiones, en muchos casos con utilización de armas blancas o de fuego. Las graves alteraciones de conducta se hacen sentir también con fuerza en los servicios asistenciales y los clínicos observamos con gran preocupación como crecen día a día los episodios conflictivos asociados al uso de cocaína entre nuestros enfermos. Los daños no afectan exclusivamente una población marginal, sino a distintos estratos de la sociedad, pasando muchas veces inadvertidos o no registrados. La coordinación entre distintas instituciones y recursos asistenciales es vital para obtener todos estos datos y proporcionar de este modo un acercamiento más riguroso a las consecuencias del consumo de la cocaína, que hoy en día estamos muy lejos de haber cuantificado en su dimensión real.

Palabras clave: cocaína, mortalidad, sobredosis, prevalencia.

\section{ABSTRACT}

According to the European Drug and Drug Addiction Observatory, after Cannabis, Cocaine is the most widely used illegal drug in Europe. Awareness of the damage caused by consuming this substance is clear in some areas, while in others it may be ignored, underestimated or poorly dimensioned. The information systems that do exist almost certainly underestimate the number of deaths connected with cocaine. Apart from the potential the drug has to cause direct damage to different organs of the body, its consumption can lead to serious problems arising from other circumstances that are not always connected with this drug, including murders, traffic accidents and accidents at work, infectious diseases related to high-risk behaviour, and suicides. Cocaine is increasingly present in cases of violence such as fights or aggressions, in many cases accompanied by the use of knives and firearms. Serious behavioural disorders are being seen more and more frequently in the healthcare services, and in clinics we are witnessing a worrying increase on a day-to-day basis of conflictive episodes associated with the use of cocaine amongst our patients. This damage does not only affect a marginal population, but instead all levels of society, and often goes unnoticed or unreported. Coordination between different institutions and healthcare areas is essential in order to obtain this information, and in this way to provide a more precise picture of the consequences of Cocaine consumption, as today we are still far from having quantified its true dimension.

Key words: cocaine, mortality, overdose, prevalence. egún ha publicado el Observatorio Europeo de las Drogas y Toxicomanías (OEDT) en su memoria del año $2007^{1}$ la cocaína es, después del cannabis, la droga ilegal más consumida en Europa, si bien estos datos muestran una importante variación entre los países que forman la Unión. Se calcula que han consumido cocaína al menos una vez (prevalencia de vida) más de 12 millones de europeos (4\% de la población adulta) de los cuales 7,5 millones son jóvenes de 15 a 34 años. El OEDT destaca que en los últimos años se ha detectado un incremento considerable del consumo, con especial relevancia en determi- 
nados entornos o escenarios (entre jóvenes, locales de diversión nocturna, etc.). También se ha hecho especialmente notable entre consumidores de opiáceos, en muchas ocasiones incluidos en programas de tratamiento sustitutivo con metadona o buprenorfina. Aunque la media de la Unión Europea es claramente inferior, si se atiende al consumo entre los jóvenes durante el último año, la prevalencia para algunos países como España $(5,2 \%)$ y el Reino Unido $(4,9 \%)$ es incluso superior a la de Estados Unidos (4,8\%). Estas prevalencias son claramente preocupantes, ya que se han incrementado en pocos años, equiparándose a las existentes en países que tradicionalmente mostraron elevados consumos, como Estados Unidos, en los que daños graves asociados al consumo de esta sustancia se hacían notar desde hace tiempo.

Las consecuencias del incremento en el uso de cocaína se pueden observar claramente a varios niveles. De un lado, la extensión del consumo se ha hecho notar de forma muy importante en las solicitudes de tratamiento, especialmente en países como España o los Países Bajos, aunque a nivel de toda la UE supuso tan solo el $13 \%$ de todas las solicitudes en 2005 . En nuestro entorno, la Comunidad Autónoma de Galicia, para los pacientes que inician tratamiento por primera vez, la cocaína como droga que motiva la asistencia supone ya más del $50 \%$ de los casos, duplicando las demandas relacionadas con el consumo de opiáceos. Se constata de este modo que la relación con la cocaína ha determinado que muchos consumidores acaben desarrollando una fuerte adicción a esta sustancia para la que lamentablemente carecemos de tratamientos farmacológicos exitosos. Además, está bien establecido que los pacientes tratados por consumo de opiáceos pueden desestabilizarse a consecuencia del consumo simultáneo de cocaína y los datos proporcionados por los clínicos no dejan lugar a dudas sobre las graves dificultades que se están observando en pacientes tratados con metadona que inician el consumo de cocaína.

Una de las consecuencias más graves del consumo de cocaína es su capacidad para condicionar un incremento de la mortalidad directa o indirecta que pensamos puede estar, en el momento actual, insuficientemente valorada o infraestimada y que es preciso investigar en mayor profundidad para conocer su magnitud ya que, si atendemos únicamente a la mortalidad comunicada directamente a los sistemas de información, por las denominadas muertes por reacción tras consumo de drogas, podrían quedar fuera de los registros muchas otras muertes relacionadas con el uso de de la cocaína ${ }^{2,3}$. Pero además de la capacidad que la cocaína tiene para producir daños directos en distintos órganos, el consumo de esta droga puede condicionar un incremento de la mortalidad entre los consumidores a través de otras circunstancias: homicidios, accidentes de tráfico y laborales, adquisición de enfermedades infecciosas relacionadas con conductas de riesgo, suicidios, etc. En este sentido pensamos que de los posibles daños que puede producir la cocaína solo accedemos a ver la punta del iceberg.

En lo que atañe a las denominadas reacciones agudas tras el consumo de drogas, en el año 2005, se comunicaron más de 400 muertes relacionadas con la cocaína en Europa aunque, los datos son todavía muy limitados en función de las deficiencias que presentan los registros de información. Ahora bien, las muertes por sobredosis debidas a la cocaína son relativamente raras y difíciles de determinar, salvo en casos de exposición masiva ${ }^{4}$. También es cierto que aunque existe una tendencia en hablar de muertes relacionadas con el consumo de heroína o sobredosis de opiáceos, desde hace años sabemos a ciencia cierta que la mayoría de las reacciones agudas a drogas se producen en presencia de policonsumo y el papel que juega la cocaína cada vez es mayor. Está claramente probado que, en presencia de cocaína, las dosis de opiáceos observadas en sujetos que fallecen son significativamente menores que cuando el consumo es exclusivamente de opiáceos ${ }^{\mathbf{5}}$, ¿a que droga atribuir la causa del fallecimiento en estos casos?

Sabemos con certeza que, a menudo, las muertes relacionadas con la cocaína pueden deberse al daño crónico que esta sustancia determina a nivel cardiovascular o por las complicaciones neurológicas que provoca. Investigaciones realizadas en Australia muestran como los niveles de patología cardíaca o cerebrovascular severas son mucho mayores en sujetos que fallecieron y que consumían cocaína frente a otros que consumían opiáceos o grupos control (suicidas) con similares características demográficas. Algunos de los daños potencialmente causados por la cocaína como hipertrofia ventricular, enfermedad isquémica cardíaca, aterosclerosis cerebrovascular, entre otras patologías, se detectan de forma significativamente mayor en los consumidores de cocaína ${ }^{6}$.

Como para los opiáceos, las variaciones en las dosis que pueden provocar muerte están muy relacionadas con factores individuales y con la capacidad para desarrollar tolerancia farmacológica y la presencia de otras enfermedades preexistentes en muchos casos no conocidas o de ciertos factores que producen una especial vulnerabilidad y proporcionan un campo abonado para que el consumo de cocaína determine fatales consecuencias. Algunas evidencias señalan una predilección genética para la susceptibilidad tóxica de la cocaína en relación con defectos en mecanismos cerebrales de la termorregulación. Determinadas condiciones a nivel receptorial en las vías estriatales dopaminérgicas o en los canales iónicos podrían influir en que sujetos más proclives a fallecer de modo súbito7, 8. Del mismo modo se ha postulado que las elevadas temperaturas ambientales podrían condicionar mayor presencia de episodios fatales asociados al consumo 
de cocaína ${ }^{9}$. En cualquier caso, la interpretación de los resultados de las investigaciones realizadas en casos de sujetos fallecidos ha de realizarse con extremada cautela, tanto en lo que atañe a los niveles plasmáticos de las drogas detectadas como a la atribución de la causa probable de la mortalidad por las mismas ${ }^{10}$.

La vía de administración de las sustancias es un factor a tener en cuenta pero conviene recordar que las reacciones agudas no se producen exclusivamente asociadas al consumo intravenoso, de modo que otras vías como la inhalatoria, comúnmente usada para la cocaína, que posibilita una rápida e intensa llegada de droga al cerebro pueden condicionar funestos daños.

Distintos estudios, relacionados con la mortalidad en accidentes de tráfico, nos muestran como sujetos que fallecen en relación con la conducción de vehículos de motor habían consumido cocaína momentos inmediatamente previos a los de producirse la muerte. Sin embargo, en algunos países, en muchos casos, esos fallecimientos se comunican como accidentes de tráfico y no se declaran como muertes relacionadas con el consumo de cocaína. En relación con esto y según los últimos datos publicados por el Instituto Nacional de Toxicología relativos a los accidentes de tráfico producidos en el año 2003 en España, revelan que en un $5,8 \%$ del total de los conductores fallecidos en ese año (1447) el consumo de alcohol etílico fue asociado con el consumo de alguna droga de abuso, siendo la combinación con la cocaína $(2,7 \%)$ la más importante, seguida del cannabis $(1,3 \%)$ y los derivados anfetamínicos $(0,6 \%)$. Pero hay que destacar además, que en 79 de los conductores fallecidos el alcohol etílico fue negativo mientras que la presencia de cannabis, cocaína y derivados anfetamínicos fue detectada $(1,5 \%, 1 \% \text { y } 0,6 \% \text { respectivamente) })^{\mathbf{1 1}}$.

Datos de algunos estudios de cohortes de pacientes consumidores de cocaína-crack nos muestran que la mortalidad en estos grupos es muy elevada y en su mayoría debida a causas no naturales. Resultados de un estudio brasileño ${ }^{12}$ publicado recientemente señalan que la mortalidad relacionada con la sobredosis se aproxima al 9\% del total de muertes estudiadas para un grupo seguido entre 1992 y 1999 con una media de seguimiento de menos de 3 años. En total, un $17.9 \%$ de los 131 pacientes que integraron el grupo estudiado fallecieron y la primera causa de muerte fue atribuida a muertes violentas relacionadas con armas de fuego o de otro tipo. Se trata de una elevada mortalidad probablemente condicionada en alguna medida por el perfil de los pacientes que integraron el estudio y que se trataba de adictos a crack de características muy marginales, a menudo implicados en actividades delictivas y que residían en lugares con una elevada tasa de grave violencia asociada a este hecho.

Aunque alejados de la situación descrita, de nuevo informes aislados en nuestro país aportan datos en una dirección similar que debería preocuparnos. La presencia de la cocaína, asociada o no a otras drogas, está cada vez más presente en episodios de violencia tales como peleas o agresiones, en muchos casos con utilización de armas blancas o de fuego. Las graves alteraciones de conducta se hacen sentir también con fuerza en los servicios asistenciales y los clínicos observamos con gran preocupación como crecen día a día los episodios conflictivos asociados al uso de cocaína entre nuestros enfermos adictos que consumen cocaína. Mientras que prácticamente ha desaparecido de las consultas o de los servicios de urgencia la figura del adicto a opiáceos agitado bajo los efectos del síndrome de abstinencia, cada vez toma más fuerza la del consumidor de cocaína con conductas violentas que se ve implicado en toda suerte de episodios que causa gran malestar entre los profesionales sanitarios, otros enfermos y la comunidad en general.

Los resultados de los análisis que realizamos en nuestros laboratorios de toxicología son, sin lugar a dudas, un fiel reflejo de los cambios en los hábitos de consumo que en los últimos años se han venido produciendo en nuestro país y de forma más precisa en Galicia. Desde hace años de forma sistemática monitorizamos la mortalidad por reacción adversa al consumo de sustancias psicoactivas, así como las analíticas de los drogodependientes que pasan a disposición judicial. No es necesario profundizar demasiado en los resultados analíticos obtenidos durante este período, para darnos cuenta como han variado los patrones de consumo y como la heroína, responsable del mayor número de muertes y detenciones producidas en Galicia hasta hace unos años, ha sido desplazada con fuerza por la cocaína en la última década. Así mientras en 1994 el número de muertes producidas por el consumo de cocaína, sola o asociada a la heroína fundamentalmente, representaba un 19,5\% del total de las muertes producidas en Galicia, en 1998 este porcentaje llegó a representar el $41 \%$ de los casos. Y más aún, en 1999, por primera vez, el número de fallecimientos atribuidos al consumo de cocaína supone el $62 \%$ del total. El máximo de este auge se alcanza en el año 2004, donde la cocaína sola es considerada sustancia principal detectada en un $45 \%$ de las muertes ocurridas ese año en Galicia ${ }^{\mathbf{1 3}}$. En los últimos años esta tendencia parece estabilizarse un poco, con el repunte que comienza a observarse en el consumo de heroína.

Algo similar ha ocurrido en la población de drogodependientes que han pasado a disposición judicial durante los últimos quince años en Galicia. Mientras en los primeros años de monitorización los detenidos eran fundamentalmente consumidores habituales de heroína, en los últimos años el patrón ha cambiado radicalmente, representando los delitos relacionados con el consumo de cocaína el $80 \%$ de los casos estudiados. 
A esto tendríamos que añadir los resultados analíticos que obtenemos de pericias privadas en relación con el consumo de sustancias de abuso en menores, donde a día de hoy, la cocaína, después del cannabis es la sustancia mayoritariamente detectada.

Cuando hablamos de daños relacionados con el consumo de cocaína, son reveladores los resultados obtenidos en la última línea de investigación que hemos iniciado hace tres años sobre la detección precoz del consumo de sustancias de abuso en mujeres durante el período de gestación. Si bien podríamos pensar a priori que la aparición del síndrome de abstinencia a opiáceos en el recién nacido sería el principal problema encontrado, hoy en día, se han comenzado a observar otro tipo de patologías, alguna de ellas manifestadas tardíamente, en las que la cocaína es la principal responsable y cuyo diagnóstico precoz es de vital importancia ${ }^{\mathbf{1 4}}$. A este respecto, un estudio desarrollado que desarrollamos recientemente, basado en el análisis del vello púbico materno y el pelo del neonato, sobre muestras recogidas en los servicios de Obstetricia y Neonatología del Hospital Xeral Cies de Vigo ha demostrado la elevada incidencia del consumo de cocaína en mujeres en período de gestación. Así un 8\% de un grupo de 60 mujeres de las que no se conocían antecedentes de drogadicción resultaron positivas a la detección de cocaína, porcentaje que

\section{REFERENCIAS}

1. Observatorio Europeo de las Drogas y las Toxicomanías. Informe anual 2007: el problema de la drogodependencia en Europa. Luxemburgo: Oficina de Publicaciones Oficiales de las Comunidades Europeas. 2007.

2. Pereiro C, Bermejo A, López B. Muerte por sobredosis: de la reacción aguda tras consumo de opiáceos a la muerte asociada al policonsumo. Adicciones 2005; 17 Supl 2: 151-165.

3. Brugal MT, Rodríguez-Martos A. Morbimortalidad asociada al consumo de drogas. Adicciones 2006; 18: 9-20.

4. EMCDDA. Cocaine and crack cocaine: a growing public health issue. Luxembourg: Office for Official Publications of the European Communities. 2007

5. Pereiro C, Bermejo A, Fernández P, Tabernero MJ. Deaths from drug abuse in northwestern Spain, 1992 - 97. Addict Biol 2003; 8: 89-95.

6. Darke S, Kaye S, Duflou J. Comparative cardiac pathology among deaths due to cocaine toxicity, opioid toxicity and non-drug-related causes. Addiction 2006; 101: 1771-1777

7. Stephens BD, Jentzen JM, Karch S, Mash DC, Wetli CV. Criteria for the Interpretation of Cocaine Levels in Human Biological Samples and Their Relation to the Cause of Death. Am J Forensic Med Pathol 2004; 25: 1-10.

8. Steven B. Karch, MD. Cocaine Cardiovascular Toxicity. Southern Medical Journal 2005; 98: 794-799. llegó a alcanzar el 26 \% en aquellas donde existían antecedentes previos de consumo de sustancias psicoactivas y en el que además los neonatos presentaron algún tipo de patología, fundamentalmente síndrome de abstinencia, retardo en el crecimiento intrauterino y disminución del perímetro craneal. Se confirman una vez más la relación causal del consumo de esta sustancia con patologías perinatales, como ya reflejaron diversos autores ${ }^{15-17}$.

Estamos pues a día de hoy ante una problemática multifactorial relacionada con el consumo de cocaína. Por los datos que disponemos sabemos que se puede iniciar desde edades muy tempranas, con posibilidad de causar daños en distintos momentos de la vida de una persona e incluso participando en el fallecimiento de forma aislada o en relación con otras causas. El posible daño no afecta exclusivamente una población marginal, la de los consumidores, sino que puede afectar a distintos estratos de la sociedad, pasando muchas veces inadvertido o no registrado. La coordinación entre distintas instituciones y recursos asistenciales es vital para obtener todos estos datos y proporcionar de este modo un acercamiento más riguroso a las consecuencias del consumo de la cocaína, que hoy en día estamos muy lejos de haber cuantificado en su dimensión real.

9. Marzuk PM, Tardiff K, Leon AC, Hirsch CS, Portera L, Iqbal MI, Nock MK, Hartwell M. Ambient Temperature and Mortality From Unintentional Cocaine Overdose. JAMA 1998; 279: 1795-1800.

10. Pereiro C. Muerte por reacción aguda tras consumo de drogas en Galicia (1992-1997). Edit. Universidad de Santiago, 1999.

11. Instituto Nacional de Toxicología. Memoria Análisis Toxicológico Muertes en Accidentes de Tráfico 2003. Ministerio de Justicia. 2004.

12. Ribeiro M, Dunn J, Laranjeira R, Sesso R. High mortality among young crack cocaine users in Brazil: a 5-year follow-up study. Addiction 2004, 99: 1133-1135.

13. Consellería de Sanidade. Observatorio Gallego de Drogodependencias Informe Xeral 2006. Xunta de Galicia, 2007.

14. Lumeng JC, Cabral HJ, Gannon KK, Heeren T, Frank DA. Pre-natal exposures to cocaine and alcohol and physical growth patterns to age 8 years. Neurotoxicology and Teratology 2007; 29: 446-457.

15. Addis A, Moretti ME, Ahmend SF, Einarson TR, Koren G. Fetal effects of cocaine: an updated meta-analysis. Reprod. Toxicol 2001; 15: 3341-369.

16. Chan D, Klein J, Koren G. New methods for neonatal drugs screening. Neo Reviews 2004; 4: 1-8.

17. Strano-Rossi S, Chiarotti M, Fiori A, Auriti C, Seganti G. Cocaine abuse in pregnancy: its evaluation through hair analysis of pathological new-borns. Life Science 1996; 59: 1909-1915. 